\title{
An exploratory study of ice-cube spikes
}

\author{
Charles A. KNIGHT \\ National Center for Atmospheric Research, PO Box 3000, Boulder, Colorado 80307-3000, USA \\ E-mail: knightc@ucar.edu
}

\begin{abstract}
It is well known that ice-cube spikes form by the extrusion of water at the surface of a freezing ice cube, driven by the expansion accompanying freezing. The growing spikes are water-filled ice tubes, growing at their tips as the water is expelled. This paper represents an exploration of their formation and the principles behind whether a freezing ice cube grows a spike or not. For this purpose, ice cubes are frozen in one set of conditions to observe what happens when spikes do or do not form. Spike formation requires a nearly steady-state freezing-and-extrusion process at the growing tip, in order to maintain a nearly constant diameter. Most spikes are polycrystals with special orientation relationships that evidently allow the steady state to become established. Thus the orientations of the initial crystals, formed by chance, help to determine whether a spike forms or not. The main alternative to spike formation is flooding over the upper surface of the ice. Spike formation depends upon an effectively non-zero contact angle of water on ice that impedes flooding. The onset of flooding is probably sensitive to the ice growth rate at the water surface, which depends strongly upon the crystal orientation with respect to the water surface where it contacts the crystal.
\end{abstract}

\section{INTRODUCTION}

When water is frozen in an open container, one sometimes finds a spike of ice projecting upward from the free surface. Figure 1 includes several examples of these on ice cubes grown in ice-cube trays. They have been interpreted correctly as a result of partly confined freezing, with the liquid water being extruded through a hole in the ice at the upper surface because of the $9 \%$ expansion that accompanies freezing. The water rises through a central hole in the spike, extending the ice tube by freezing at its tip (Dorsey, 1921; Bally, 1933; Blanchard, 1951; Hallett, 1960).

An ice spike could be defined as requiring a length-todiameter ratio greater than some number, but there seems little point in doing that. Here, we emphasize the most striking ice spikes that have parallel or nearly parallel sides, as showing a steady- or nearly steady-state process.

Two experimental investigations aimed directly at understanding ice-cube spikes were completed recently (Hill and others, 2004; Libbrecht and Lui, 2004), and these motivated the present study. They confirmed the 'Bally-Dorsey hypothesis' (the explanation above) and made other contributions, but failed to investigate why spikes do not always occur: Why do most ice cubes not form spikes? What are the necessary and sufficient conditions for spike formation? Since the expansion is an inexorable consequence of the freezing, what are the other manifestations of that expansion, and what selects between them?

Most basic of all, what are the important physical principles that govern ice-spike formation? This question was the main object of my curiosity, and my initial approach was simply to watch ice spikes forming, looking for what differentiates between forming and not forming spikes under the same external conditions. The investigation grew from that, and progressed to the point of having, I think, a much better appreciation of the principles than I had to start with, or is available in the literature. As will be seen, this is still short of having a specific list of necessary and sufficient conditions for spike formation, but it should be a step toward that, if or when someone pursues it.

\section{INITIAL EXPERIMENTS}

Ice cubes were frozen in a walk-in cold room by putting icecube trays with water in them on a tabletop. The room was controlled at a mean temperature of about $-15^{\circ} \mathrm{C}$, fluctuating several degrees either way, and there was a wind of several $\mathrm{m} \mathrm{s}^{-1}$. The ice-cube trays had 16 compartments; half were left empty, and the others were filled with deionized water $\left(18 \Omega \mathrm{m}\right.$ resistivity) at $22-23^{\circ} \mathrm{C}$.

Freezing at the water surface may start in 15-20 minutes, but sometimes not for 1 hour or more. When ice does initiate in these deeply supercooled samples, a fine mesh of ice dendrites grows throughout the volume in about $1 \mathrm{~s}$ (Pruppacher, 1967; Macklin and Ryan, 1968). The liquid portion of this slush then freezes from the outside. These ice cubes were never seen to develop spikes, or even any pronounced local bulges on their upper surfaces. The example in Figure $1 \mathrm{~g}$ has no bulges at all, but that is rare. The usual case has a wide, rounded dome somewhere on the top surface. Not counting these cases, still only $10-20 \%$ of the ice cubes grow spikes, so their rarity still requires an explanation.

Most of the ice initiation in the cold room is probably from tiny ice crystals in the air. Because it was desired to avoid deep supercooling in order to increase the frequency of spike formation, a little ice was sawed on the band-saw in the cold room about 15 min after cooling commenced in all subsequent experiments. Done judiciously, this initiated ice early, at several places on the surface in each ice-cube compartment, in a variety of crystal orientations. However, since this is still not a reproducible procedure in terms of the factors important for ice-spike formation, the numerical frequency of formation is still not very meaningful. Most of the subsequent investigation centered on understanding the early crystal textures, observing how they relate to ice-spike formation and explaining the relationship that was found.

The following observations were gained from watching the freezing in these initial experiments. The most common location for spike formation is the last, unfrozen area of the water surface, usually tri- or quadrangular, bounded by three 


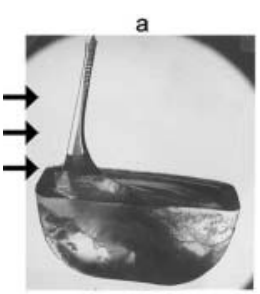

d1
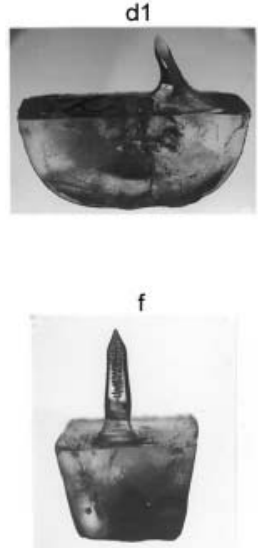

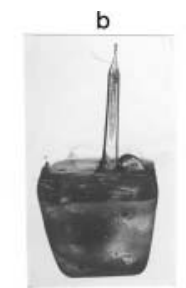

d2
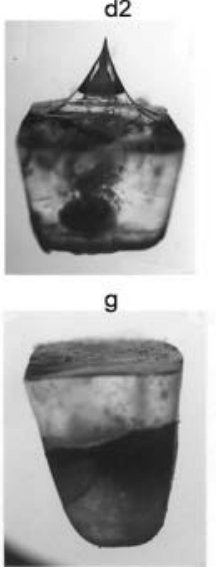
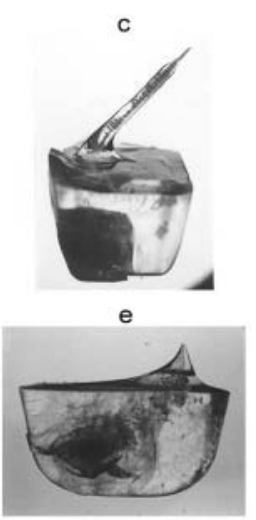

h

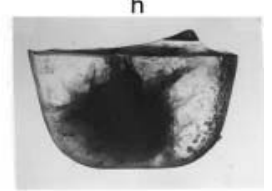

$1 \mathrm{~cm}$
Fig. 1. Examples of ice cubes, discussed in the text. The 'spikes' are seen in (a), (b), (c) and (f); protuberances that did not become spikes in (d), (e) and (h); and an ice cube with an exceptionally flat surface, from water that supercooled to at least $-10^{\circ} \mathrm{C}$ before ice initiated, in (g). Side and end views of the same cube are shown in (d1) and (d2). The changes in illumination near the bases of the spikes in (a) and (d), and see also (e) and (h), are an illumination effect and do not indicate any changes in the ice.

or four crystals. Both Hallett (1960) and Hill and others (2004) comment on this. As this opening closes down, water is squeezed upward, making a very pronounced, rounded bulge above the ice surface. Having shrunk to a diameter of a few millimeters, this bulge sometimes continues growing as a spike to as much as several centimeters in length as seen in Figure 1. Spike growth rates varied from nearly as fast as $1 \mathrm{~mm} \mathrm{~s}^{-1}$ to roughly $1 \mathrm{~mm} \mathrm{~min}^{-1}$. Growing just as the upper surface is not quite completely frozen, spikes occur very early in the freezing of the ice cubes as a whole, before the expansion has any opportunity to crack the ice, and before any air bubbles form in the liquid: they generally grow 10-40 min after the first ice forms. It took about 4 hours for the ice cubes to freeze solid.

Figure 2 illustrates the water bulging up into open spaces in the ice cover. It was taken with light reflected from the surface, with the water freezing in a circular plastic dish. The camera pointed not quite straight down on the surface, and the illumination was from a circular, diffuse source, arranged so that initially the flat, water surface was fully illuminated by reflection (Fig. 2, $t=2: 00$ (time in minutes and seconds), upper right panel). The bulges of water above the freezing surface act as convex mirrors, so the light source is imaged as bright spots in the middle of each bulge (Fig. 2, $t=2: 00$ to $t=5: 50$ ): the smaller the bright spot, the greater the curvature of the water surface.

The polygonal holes in the ice surface in which these bulges occur are bounded by surface crystals that earlier were needle-like, each with its $c$ axis in the plane normal to the needle axis. Two of them froze over between 2:00 and 5:00, but then between 5:00 and 5:50 water flowed out over the ice at the upper left, and the level of the water surface in the remaining openings sank back down. These flooding events are very common. The surface of the flood then starts to freeze inward from its perimeter, and there often ensues a series of such discrete flooding events, eventually leaving a wide, low, sometimes terraced, plateau of ice above the original ice surface. Figure 2 also shows two areas of rather uniformly textured ice, to the lower left and lower right, which are areas with the $c$ axis not far from vertical. When most of the surface is covered with this kind of ice, flooding usually occurs very early, and spikes rarely form.

The name 'ice spike' implies that they are usually quite straight, even when highly tilted, and taper very little over much of their length. Figure 1 also shows projections from the ice surface that taper to fairly sharp points and therefore are not termed spikes. Occasionally, as in Figure $1 \mathrm{~d} 1$ and $\mathrm{d} 2$, a projection looks like a spike from one direction but not from another.

The initial observations summarized above underscore the importance of two factors in ice-spike formation. One is the relationship between the crystal fabrics of the initial ice skim, the highly rounded water bulges above the surface and the eventual spikes. The other is the flooding events, since flooding is evidently the main alternative to spike formation early in the freezing. The crystal fabrics will be discussed first, followed by the formation of the first ice layer on the surface, and then the flooding events. A final discussion then concerns the steady state, and returns to the principles involved in understanding why spikes are rather rare.

\section{CRYSTAL FABRICS OF THE ICE-CUBE SPIKES}

With the exception of brief observations by Hallett (1960) the literature contains no information on crystal conformations or orientations in ice spikes. The next step in the present study was to determine these for a number of spikes. The measurements employed a 'Rigsby stage' (Langway, 1958), constructed to accept $5 \mathrm{~cm}$-square glass plates (on which the thin sections of ice were mounted), and fitted with a low-power microscope for viewing. The crystal $c$-axis orientations were measured optically, between crossed polaroids, using standard procedures (e.g. Langway, 1958).

The spikes themselves are inconveniently small to section individually, so first they were cast in solid ice. Sections were then made through the ice, parallel to the original upper surface. The spikes were almost always easily recognizable, though their edges often were not precisely defined. Their most characteristic feature is a central line of air bubbles formed when the central conduit freezes solid (see Fig. 1, especially Fig. 1c and f). The central air bubbles nearly always coincide with a grain boundary or a triple junction line along the center of the spike. Often a series of thin sections was prepared through a spike, starting at the upper surface of the built-up ice and ending just below the original flat part of the ice-cube surface. There is always perfect crystal continuity into the body of the ice cube, and no indication of recrystallization altering the crystals within a spike.

Figure $3 a-c$ show the crystal orientations of component crystals of the ice-cube spike shown in Figure 1a, looking down on the horizontal slices. The arrow in Figure 3a represents the projection of the axis of the spike, labeled with its approximate angle from the vertical, the arrow pointing from base to tip. This spike is a tricrystal, with the grain boundary intersection line approximately up the center. The orientation symbol for each crystal is the strike 

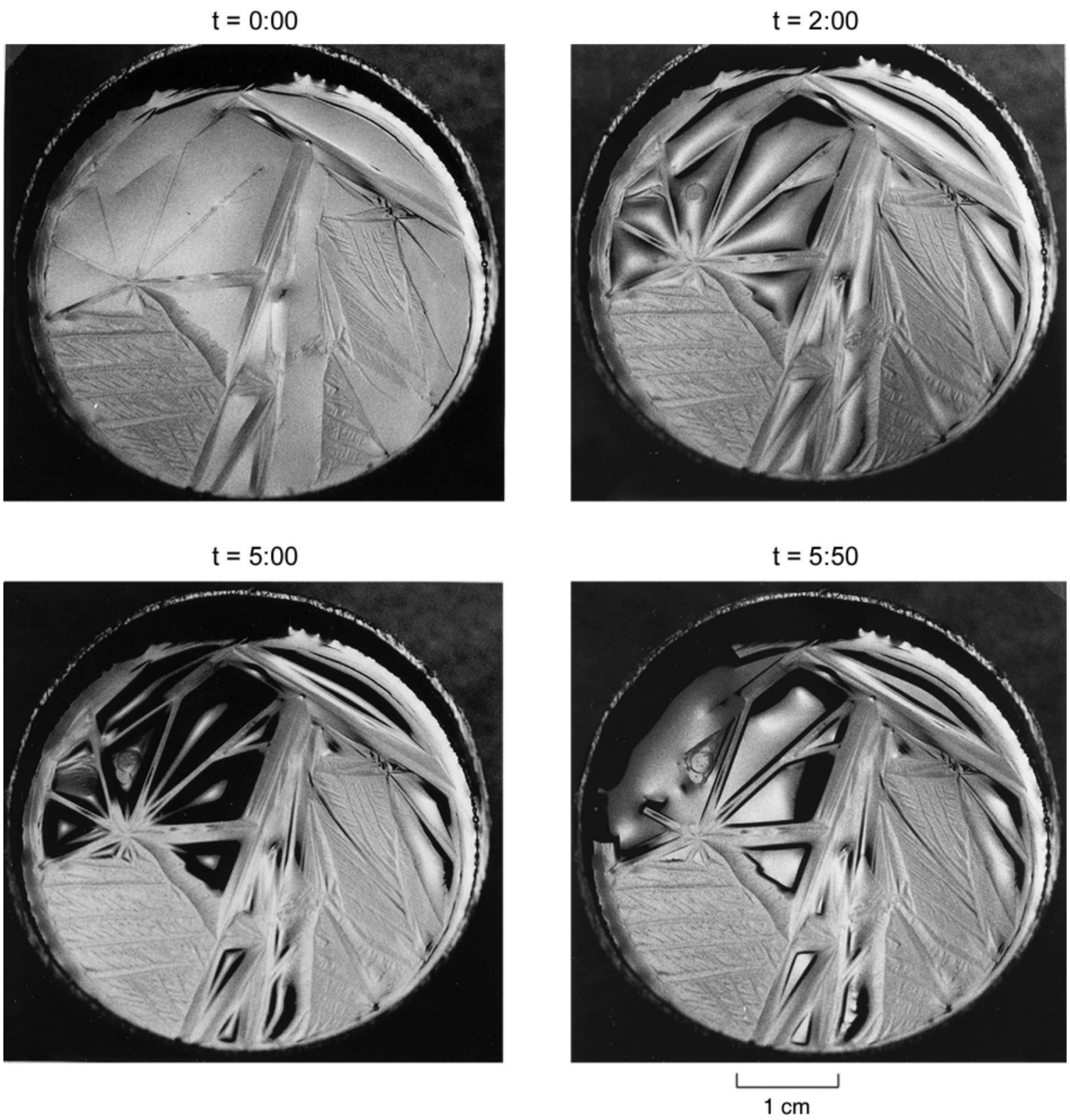

Fig. 2. Four stages in the ice growth across the water surface. Illumination is diffuse light reflected from the surface, and times are indicated in minutes and seconds. Note the water bulging up between the needle crystals at 2:00. At 5:00, two of the bulges (near the left edge) have frozen, and flooding occurred at the upper left between 5:00 and 5:50, lowering the water level in the unfrozen openings. The evenly textured areas are covered with a thin skim of ice with its $c$ axis close to vertical.

and dip of the basal plane (0001). The longer line traces the intersection of that plane with the plane of the section (the horizontal), and the shorter one normal to it is the direction of its downward tilt. This is measured in degrees from the horizontal, as labeled.

The positions of the three slices in Figure 3 are indicated by arrows on Figure 1a. Figure 3c is the lowest, close to the flat part of the original ice-cube surface. Before the sections were made, the righthand third of the ice cube was cut off. The notches are from two vertical saw cuts, to provide a better spatial registry in case the angle of the spike needed to be determined from the sections.

The lines of intersection of the basal planes with the horizontal are roughly tangential in the spike, as expected if the spike grew from a triangular opening between three surface needles. The new result is the consistent inward dip of the basal planes of ice, downward toward the center of the ice spike. Thus the basal planes diverge upwards or, for highly tilted spikes, outwards along the spike axis. Figure $3 \mathrm{~d}$ is a schematic cross-section through the center of the spike, as if it were composed only of the two crystals on the right (ignoring the one on the left in Fig. 3a-c), at about the orientation of the dashed line in Figure 3c. The tilt of the ice spike has it growing at an angle that roughly bisects the angle between the two sets of basal planes, as shown in Figure $3 \mathrm{~d}$. These two features are general rules for the ice spikes studied: the upward divergence of the basal planes of the component crystals of the spike (whose basal planes are roughly tangential in the spike itself) and their relationship with the tilt of the spike. 


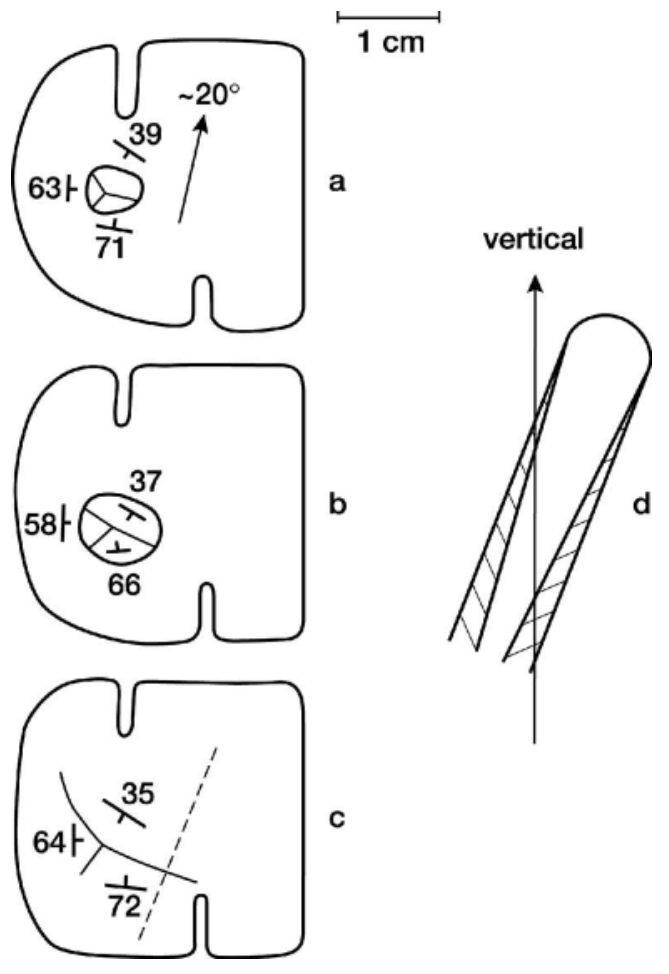

Fig. 3. $(\mathrm{a}-\mathrm{c})$ Crystal orientations of the three crystals composing the spike in Figure 1a, at three levels: (a), (b) and (c) correspond to the three arrows, starting at the top, in Figure 1a. The numbers are the inclination of the basal planes from horizontal, and the symbols show the strike and dip of the basal planes, explained in the text. The outlines are the edge of the built-up ice; the notches are vertical saw cuts to provide a registration between successive sections. The spike slants about $20^{\circ}$ from the vertical, indicated by the arrow in (a). (d) A schematic cross-section of the growing spike, showing the water core and the ice shell, in the plane of its inclination (the dashed line in (c)) and about normal to the boundary between the two major crystals, whose basal plane orientations are indicated by the parallel lines.

Figure 4 contains more ice fabrics, Figure $4 a-e$ being from the ice spikes in Figure $1 \mathrm{~b}$ and $\mathrm{c}$ and the ice projections in Figure $1 \mathrm{~d}$, e and $\mathrm{h}$, respectively. The ice spike of Figure $1 \mathrm{f}$ is not included since it is a single crystal, with its $C$ axis vertical, underlain by a crystal of the same orientation. Three such spikes, two of which were vertical, were encountered, of the 34 whose crystal orientations were recorded. (The one that was slightly tilted leaned downwind.) All of the ice cubes in Figure 4 were trimmed before sectioning, so as to fit comfortably on the $5 \times 5 \mathrm{~cm}$ glass slides, and the sections for Figure $4 d$ and e were right at the original ice surface.

Figure $4 \mathrm{a}$ is a section through the spike in Figure $1 \mathrm{~b}$, several millimeters above the ice-cube surface. This one is a bicrystal, tilting only $10^{\circ}$ from the vertical, with its two crystals' basal planes diverging symmetrically upward. Figure $4 \mathrm{~b}$ (Fig. 1c) is a case of extreme tilt, again roughly bisecting the angle between the basal planes of its two component crystals. Figure 4c contains two levels in the ice protrusion seen in two views in Figure $1 \mathrm{~d} 1$ and $\mathrm{d} 2$. One might call this an ice spike, looking only at the side of the ice cube, but not looking from the end. This one started out as four crystals, with its initial tilt consistent with the asymmetry of the tilts of the basal planes, but ended up as two crystals with the basal planes $<30^{\circ}$ apart. The crystal orientations in two other ice projections that did not grow a

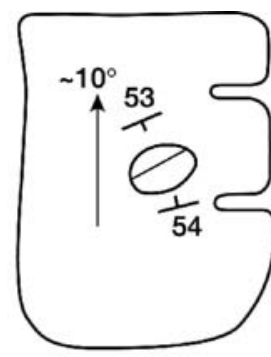

c1

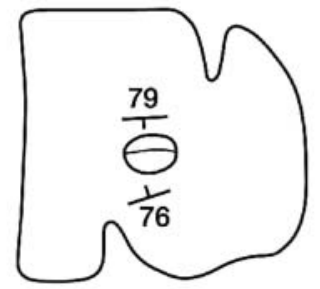

d

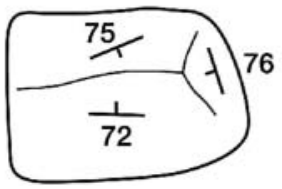

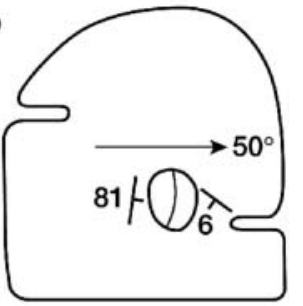

c2

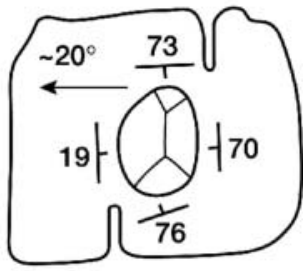

e

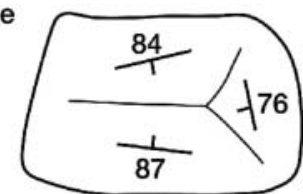

Fig. 4. Crystal orientations from ice cubes in Figure 1: (a) is from Figure 1b; (b) from Figure 1c; (c1) and (c2) from Figure 1d1 and 1d2; (d) from Figure 1e; and (e) from Figure 1f. ((c2) is a horizontal section near the base of the spike shown in Figure $1 \mathrm{~d} 1$ and $\mathrm{d} 2$, and (c1) is higher up, above where two of the crystals had terminated at the sides of the ice.)

into spikes (Fig. 1e and h) are shown in Figure 4d and e. From comparison with the true spikes given in Figures 3 and $4 \mathrm{a}$ and $\mathrm{b}$, it appears that considerable upward divergence of the basal planes is needed to produce the long ice spikes. Shorter, tapering ice projections form when the divergence is not great enough, and no projections of this kind are seen when the basal planes converge upwards.

This is one general result from the fabric studies, less than $20 \%$ of which have been illustrated. Others are that the crystal orientations control the spike orientations, and spikes with either two or three crystals are by far the most common, one crystal and four or more crystals being very rare.

\section{FREEZING AT A FREE WATER SURFACE: HOW MANY OF THE SPIKES GET STARTED?}

An ice single crystal growing in slightly supercooled water has extremely anisotropic growth rates: very slow parallel to its $c$ axis and much faster parallel to the basal plane (e.g. Hobbs, 1974). At a free water surface, this leads to thin, basal, single-crystal discs floating with their $c$ axes vertical given that they start in that orientation. Starting at some other orientation, they might reorient that way if they were free to do so. In any case, the ice initiation in the present experiments provided a variety of starting orientations that soon were locked in as crystals grew and stuck to each other and to the walls. Initiation by debris from the band-saw 
provides some free-floating single crystals, and also polycrystals that give rise to several needles radiating from one center, as seen toward the left side of Figure 2.

Hallett (1960) has described ice growth at a supercooled water surface as a function of crystal orientation. An ice crystal with its $c$ axis vertical has its fast-growth plane parallel to the surface and spreads quickly and fairly uniformly as a thin layer over the entire surface. A crystal with its $C$ axis horizontal (or at any angle not too near the vertical) grows as a needle normal to the $c$ axis, extending linearly very fast but not covering area nearly as fast. Hallett (1960) calls these 'surface needles' to distinguish them from snow crystal needles. The interlocking of these surface needles and their slow thickening produces the triangular and more-than-three-sided polygonal 'holes' of open water that slowly close in from their sides, producing the water bulges as in Figure 2.

The $c$ axes of most surface needles are not parallel to the surface, so their basal planes usually slant down to one side. Basal dendrites grow down into the water on that side, as seen in Figure 5a, which is very much like Hallett's (1960) figure 3. The water had supercooled to some depth. Hallett (1960) observed that the lateral growth at the surface was faster on the side away from these dendrites, perhaps because of the latent heat released by growth of the downward-slanting dendrites.

This slanting growth down into the liquid (illustrated in Hallett's fig. 1) was seen many times in the present experiments, and may nearly always be associated with spike formation. However, Figure 5a may not be the whole story. Since the surface bulges of water are predecessors of ice-spike formation, it is probably more relevant to draw the water surface as in Figure 5b, with the water being squeezed up above the ice. Figure $5 \mathrm{a}$ is appropriate for free-floating ice, whereas Figure 5b applies to ice fixed in place. Now, remembering that the slanting lines in the ice indicate the basal orientation, direction $\mathrm{D}$ is optimally oriented for the fastest growth along the actual tilted water surface. Growth there is parallel to the basal plane, while growth in direction $\mathrm{C}$ is perpendicular. This effect must produce a strong bias toward the last open-water space being surrounded by surface needles with the basal planes of their component crystals diverging upwards, as is observed in the ice spikes. The interaction of the changing slope of the water surface at the ice-water-air junction with the extreme anisotropy of ice growth rate in the plane of the surface is critical for icespike formation, and is discussed further in connection with Figure 9, below.

\section{SURFACE FLOODING; THE EFFECTIVE CONTACT ANGLE OF WATER ON ICE}

Watching ice cubes freeze, one quickly sees that surface flooding is the main alternative to ice-spike formation, so the most fundamental issue is why the flooding occurs in some cases but not others. The water surface commonly becomes elevated above the ice surface during early freezing, and at the tips of growing spikes the slope of the water surface at its contact with ice can be vertical or overhanging, without flooding the ice (demonstrated below). What keeps the water from flowing downhill? The effective contact angle of water on ice must be greater than zero, since if it were zero, there would be no barrier. ('Effective' is used here because the system is neither at equilibrium nor strictly pure.)

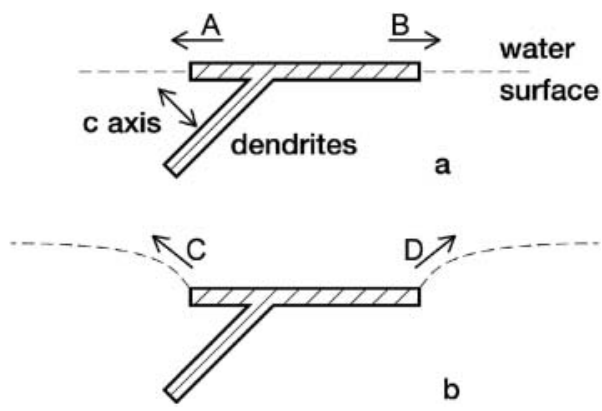

Fig. 5. (a) A cross-sectional view of how a 'surface needle' with a tilted $c$ axis spreads laterally on a water surface (after Hallett, 1960, fig. 3). The initial growth is down into the water parallel to the basal plane, and on the surface the lateral growth to the right (B) is faster than that to the left (A). (b) The same situation taking into account the rising of the water level when the freezing is confined and the growing crystal is locked in place so that it cannot float upward. Now the growth along the surface to the right $(C)$ is much faster than that to the left (D), because it is nearly parallel to the basal plane, whereas that to the left is nearly parallel to the $c$ axis.

The onset of flooding is sudden, with a rapid initial outflow that slows quickly, stops, and the surface of the puddle then freezes more slowly back (uphill) from the edge. During the flood the advancing water-ice contact line appears very sharp, but as the surface freezing moves back, there is no visible contact line in reflected light: there is no sharp change in the tilt of the upper surface.

In the process of photography it was found that the flooding is very sensitive to mechanical disturbance: sometimes the slightest vibration or shock starts it. Vibration may be another factor contributing to the rarity of ice spikes. (It is best to move the camera, not the specimen, when photographing ice-spike growth and its precursors.) A simple experiment showed that vibrating the ice-cube trays can prevent spike formation entirely, but a quantitative study of the effects of vibration was not undertaken.

Small additions of salt discourage ice-spike formation by encouraging flooding, probably not by any influence on growth at the tip of the spike caused by build-up of impurities, as was suggested by Libbrecht and Lui (2004). The effect is illustrated in Figure 6, five panels of which (all but $t=9: 29$ ) show stages of the freezing of a $10^{-2}$ molar $\mathrm{NaCl}$ solution. The pure sample, $t=9: 29$, was started at the same time and is included for comparison. Flooding over the ice starts very early with a $10^{-2}$ molar salt solution, and proceeds continuously. In the 2 min interval between Figure 6, 1:31 and 3:29, for instance, the contact line creeps steadily over the ice. In contrast, the pure water in this case flooded a little, once, very early, and then never again. Instead, it grew a thick ice spike, about $1 \mathrm{~cm}$ high when photographed, that grew later to $>2 \mathrm{~cm}$. Experiments with $10^{-3}$ molar $\mathrm{NaCl}$ showed similar behavior to $10^{-2}$ molar, in that, once started, the flooding tended to continue, only a lot more slowly. Before it eventually started, however, much more prominent water bulges developed, and in one case a small ice spike formed. The slow motion of the contact line over the ice in the salty cases might largely be a result of evaporation at the contact line, concentrating salt in the water there and lowering the melting point, perhaps causing the contact line to advance by local melting. However, the explanation of the effect of salt on flooding was not pursued. The flooding that 


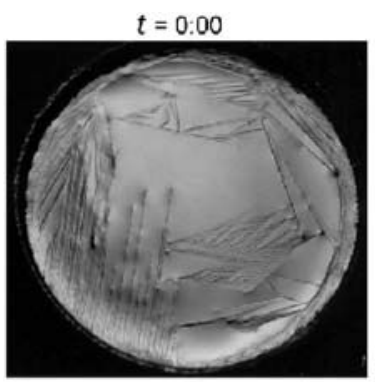

$t=3: 29$

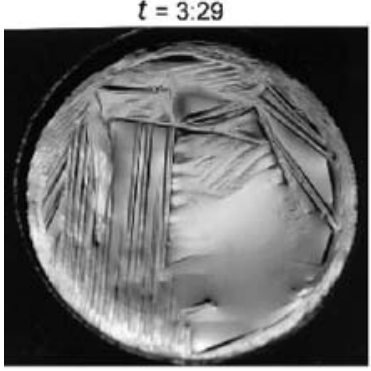

$t=10: 27$

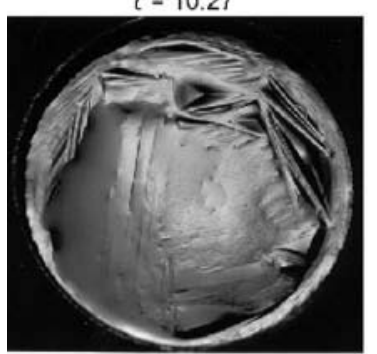

$1 \mathrm{~cm}$

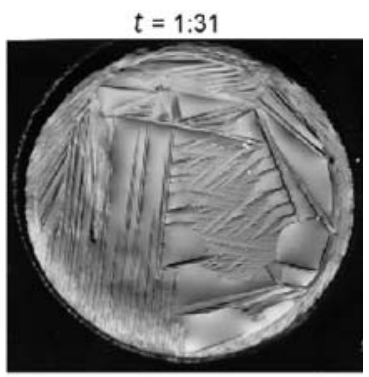

$t=9: 29$

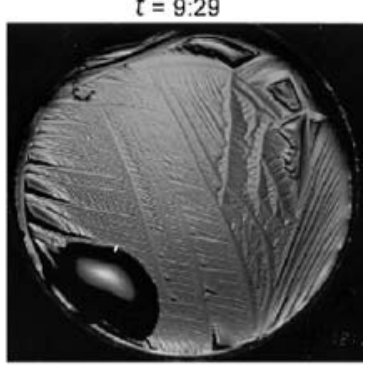

$\ell=29: 26$

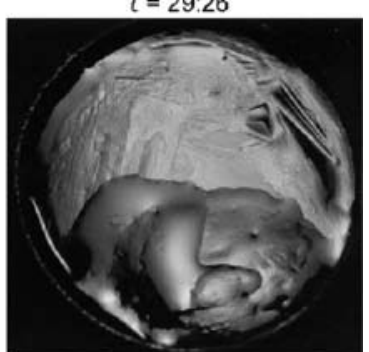

Fig. 6. Sequence similar to Figure 2, but showing the growth and flooding of the ice from a $10^{-2}$ molar $\mathrm{NaCl}$ solution, except for the one at $t=9: 29$ (minutes and seconds after 0:00), which is from pure water. The flooding from the $\mathrm{NaCl}$ solution starts early and proceeds continuously. Gradual flooding prevails with the salt solution, but with pure water there was very limited flooding and an ice spike grew instead.

often occurs with pure water, as seen in Figure 2, is less extensive and more discontinuous but can lead to terraced ice hillocks not unlike that seen in Figure 6, $t=29: 26$.

\section{CONCLUDING DISCUSSIONS}

\subsection{Principles of ice-spike formation: achieving and maintaining the balance required for steady-state growth}

For a spike to have very nearly parallel sides, which the longest do for most of their length in the growth conditions used here, the tip must be essentially identical from one moment to the next. The ice front must move at the same rate as the water front, or else the diameter of the spike will change. A closer look at a single spike illustrates this. Figure 7 is a series of photographs of a growing spike that maintains a constant diameter for about $1 \mathrm{~cm}$. The tip outline over most of its growth is a virtually perfect half-circle, and there is no drainage of water down the sides. The axial growth rate is constant for about $10 \mathrm{~min}$ (Fig. 8). Then it slows, probably when the main water supply is cut off by freezing at the base of the spike; the diameter decreases, and the axial growth rate speeds up again as the last of the water within the spike freezes (Hill and others, 2004). (Note that the camera had to be raised at about $10 \mathrm{~min}$, and the curves before and after that were lined up.)
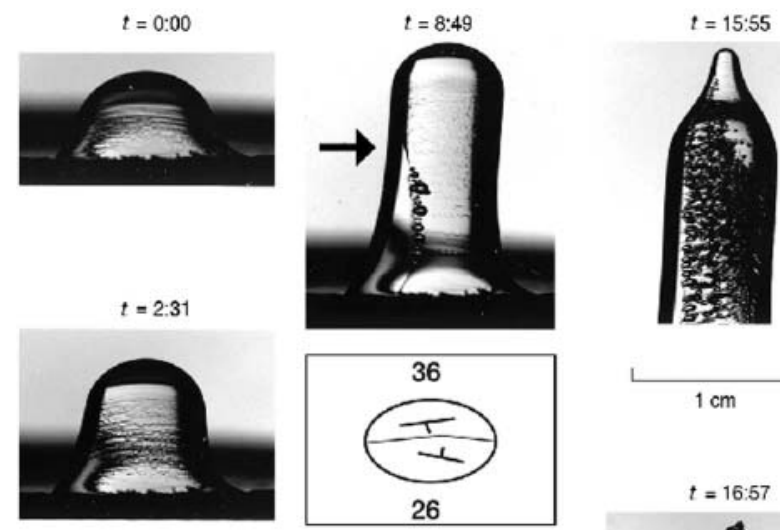

$t=5: 22$

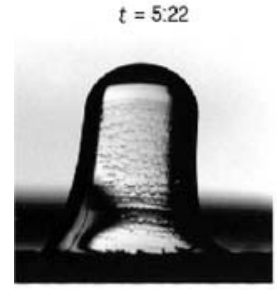

$t=12: 3$
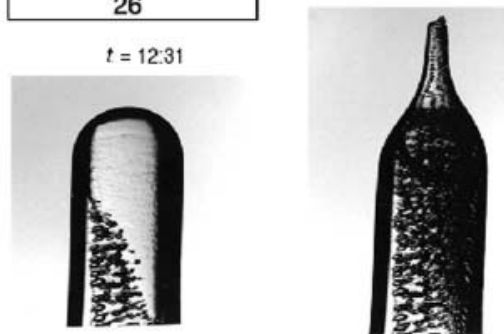

Fig. 7. Close-up series of a growing spike, with times in minutes and seconds. After 8:49, the camera was moved upward to keep the tip in view: the arrow indicates the location of the bottom of the last three views. The spike was composed of two crystals, with orientations given in the center, oriented such that looking from below on the page is the viewpoint of the photographs.

The motion of the junction line where the phases water, ice and air meet is important when considering ice-spike growth. This line (a point on two-dimensional diagrams) moves because of the freezing of the water. The fact that there is no break in the slope of the surface-air interface of the condensed phases at the water-ice junction shows that the junction follows the water-air interface. This is not necessarily true a priori, but is an observed fact, and it is critically important for the reasoning that follows. The junction always follows the water surface (except of course when water is flooding over the ice), even as the water surface itself is moving; the motion of the junction does not drag the water surface with it.

Thus the junction itself is not visible on photos like those in Figure 7. However, in steady-state growth, when a spike has parallel sides, it must be just where the tip curvature starts, because the water-air interface at the junction has to be parallel to the axis of the spike for the spike diameter to remain constant. The liquid cap then has to be a half-circle in cross-section, neglecting gravity. (This is the case in Figure 7 when the sides are parallel, and Figure 10, below, is a direct demonstration of the location of the junction for a similar spike.) The 'axial crystal growth rate' of the ice, which is the rate of movement of the junction in the axial direction, must equal the 'axial extrusion rate' of the water, defined as the volume extrusion rate divided by the spike cross-sectional area, if the sides of the spike are to be parallel.

These two axial rates are somewhat independent, inasmuch as the former depends upon the heat economy and the crystal orientations at the tip of the spike, while the latter depends upon the freezing rate of the body of the ice cube and the cross-sectional area of the spike. However, given an approximately constant volume extrusion rate, there probably always exists a size of opening for which the 


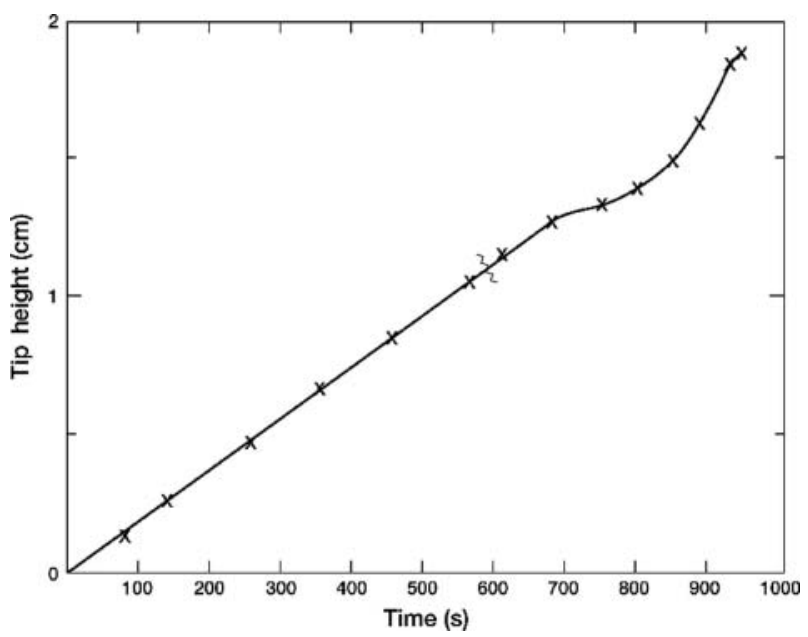

Fig. 8. Positions of the tip of the spike in Figure 7, taken from the photographs, are plotted as a function of time. The camera was moved just before $600 \mathrm{~s}$ and the two portions of the graph were lined up.

equality can hold, since the axial extrusion rate for a large opening is very slow, and can be as fast as might be needed for a small enough opening. Given that the junction follows the water surface, if there is too much water at the tip the diameter of the spike will increase because the liquid overhangs the side, and the reverse effect is seen if there is too little water at the tip. A constant rate of water extrusion will produce a parallel-sided spike; a changing rate will produce a tapering or expanding spike.

Figure 9 is a two-dimensional sketch of the history of an ice spike. It starts as an opening in the initial ice cover, in which the water surface is essentially flat. As the ice grows the opening closes in and the water bulges upward, as seen in Figure 9a. Further growth constricts the opening more, increasing the axial extrusion rate and increasing the curvature of the water surface (Fig. 9b). If a spike is to form (as in Figs 7 and 9), the axial crystal growth rate must match the axial extrusion rate just when the water-air interface becomes parallel to the spike axis. At first, there is not enough water to give a hemispherical cap, so the diameter of the opening narrows until it is hemispherical, at which point the spike continues to grow with parallel sides.

Figure 10 illustrates the principle of the adjustment of a spike to its steady-state diameter. The growing spike in Figure 10, $t=0: 00$, had its tip drop of water blown away by a blast of air from a syringe, and in the panel taken $14 \mathrm{~s}$ later the water had separated entirely, without wetting the side of the spike. There was immediately a little inward freezing, constricting the opening somewhat. The water then extruded out from that smaller opening, but soon regained about the same diameter it had before, as shown at $t=1: 55$. Figure 10 also illustrates that the water-ice junction is right where the tip curvature starts; and it also shows that the junction is usually not simply a circle around the spike. The upward arc at the tip at $t=0: 14$ is the ice edge of the nearer side of the tip. Evidently the grain boundaries occupy significant dips, probably downward cusps, in the junction line that encircles the tip.

\subsection{Influence of the crystal orientations}

The experimental results demonstrate that crystal orientations are important in determining whether ice spikes form a
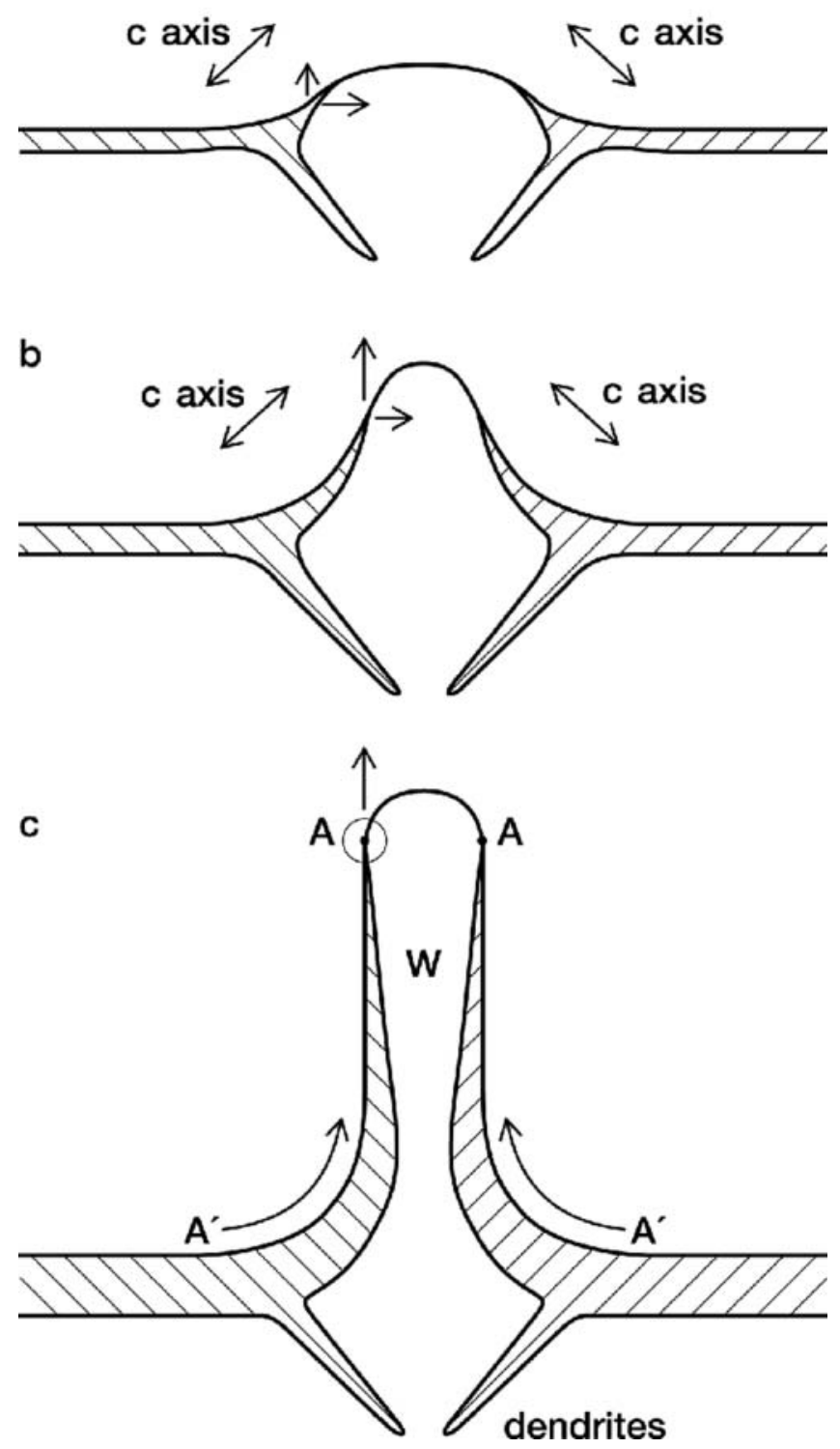

Fig. 9. $(a-c)$ Schematic of the stages of growth of a vertical ice spike like that in Figure 7 . The dendrites projecting down into the water are as in Figure 5, and have been observed directly. In (c) the motion of the ice-water-air junction is indicated, as traveling from $A^{\prime}$ to $A$. There is no horizontal motion of the junction in the steady state.

or not, and it is most likely that this importance is through the growth rate anisotropy. We attempt here to combine the effects of growth anisotropy with the steady-state discussion of section 6.1. Lacking a quantitative model of spike formation, and lacking also the information that one needs to employ such a model, such as quantitative ice growth rates at a free water surface as a function of supercooling and crystal orientation, all that can be done is to attempt to reach a reasonable, qualitative understanding.

The two-dimensional case sketched in Figure 9 is drawn with the orientations of the two crystals especially favorable to forming a vertical spike, according to all the measurements. The basal planes of the two (their fast-growth directions) diverge upwards, intersecting at about $90^{\circ}$. As the junction moves from $A^{\prime}$ to $A$ in Figure $9 c$, the ice growth direction at the junction changes from horizontal to vertical and the crystal orientation of growth along the water surface 


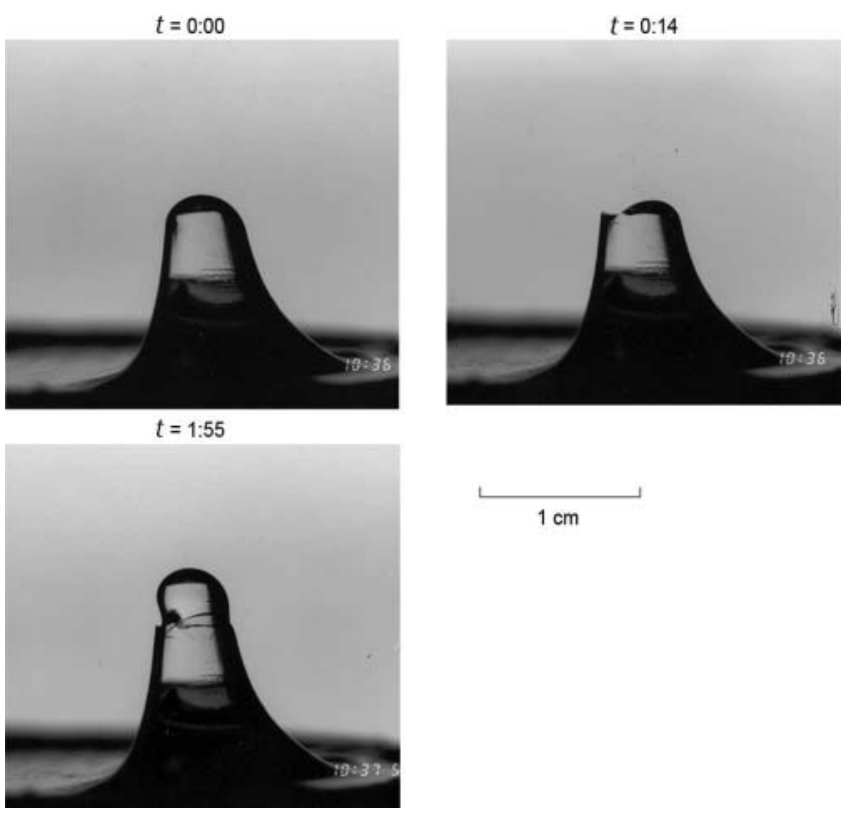

Fig. 10. Three stages of a growing spike, times in minutes and seconds. The water drop was blown off the tip with a blast of air from a syringe, between 0:00 and 0:14. The growing spike has regained its previous diameter at 1:55.

changes from $45^{\circ}$ to the $c$ axis, to parallel to the $c$ axis, and back to $45^{\circ}$ again but in the opposite sense. Correspondingly, the growth rate at the junction changes from some value directed horizontal, to a minimum value directed at $45^{\circ}$, and then it increases again and finally becomes entirely vertical. According to the discussion accompanying Figure $5 \mathrm{a}$, if the supercooling at the junction remains the same throughout, the initial, horizontal growth rate could be quite a lot slower than the final, vertical growth rate.

Why is this particular arrangement so conducive to icespike formation? What would happen if the basal planes converged instead of diverging? Consider the growth rate of ice along the water/air interface, as the orientation of that interface at the junction changes from horizontal to vertical (A to $A^{\prime}$ in Fig. 9c). Now instead of passing through the orientation of slowest growth along the water surface (parallel to the $c$ axis), it reaches the orientation of fastest growth when the tilt of the water surface reaches about $45^{\circ}$. At this point the ice growth causes the opening in the ice surface to constrict exceptionally fast rather than exceptionally slowly, and this might lead to flooding (discussed below), or it might even lead to the opening freezing shut. Either would prevent spike formation.

An interesting case that occurs about as often as true spikes is when the basal planes of all the crystals are close to vertical. Spikes do not form then, because a steady state is not attained, as seen in Figure $1 \mathrm{~d} 2$, e and $\mathrm{h}$. These are probably 'nipped in the bud' by their supply channel freezing shut. (This happens for all the spikes, but at a later stage.) Hill and others (2004) suggested that these 'volcanic bumps' were a result of overflow, but those observed here definitely did not flow over. And, it does not seem likely that they could result from freezing shut at the tip, because the axial extrusion rate would increase almost without limit as the size of the opening at the tip approached zero. One would end up with either a very thin, long spike, or a brief, fine jet of water, or the tip would freeze over and then break.
No evidence of any of these outcomes was ever seen in these features, though the final freezing of true spikes sometimes apparently includes breakage at the tip (see Fig. 7, final panel). Finding a firm explanation for why these 'volcanic bumps' do not develop into spikes remains one of the loose ends of this exploratory study.

\subsection{Spikes observed outdoors; big spikes; hollow spikes}

Early published reports of ice spikes describe spikes that occur naturally overnight, from the freezing of water standing in pots, bird-baths or other receptacles. Bally (1933), for instance, shows a spike about $15 \mathrm{~cm}$ long, inclined about $45^{\circ}$ from the vertical, and Dorsey (1921) reports one $11 \mathrm{~cm}$ long, tapering from base to tip. These are much larger than the ice-cube spikes recorded here, and probably grow much more slowly. Hallett (1960) observed large spikes on glacial puddles partially frozen overnight. There might be significant differences between the orientations that favor the ice-cube spikes of the present study and those that favor spikes in the probably warmer and windless conditions that lead to such large spikes. Hallett (1960) reported a large tricrystal spike with a crystal texture that seems different from any seen in this study of spikes on ice cubes; and he and Perry (1995) reported hollow spikes, a very puzzling occurrence unless the unfrozen water drains out for some reason as the spike is growing.

The leaning spikes are particularly interesting if they are true spikes with parallel sides, which some of the ice-cube spikes appear to be (e.g. Fig. 1c). The leading edge of the ice shell must be shaped just right to maintain the water surface at the junction line parallel to the spike axis all the way around. It seems surprising that there even exists a shape that can provide uniform ice growth rate all along the junction line and simultaneously have all the tangents at the junction line parallel. The lower crystal or crystals of the spike must project farther than the upper one(s), to compensate for the asymmetrical deformation of the water at the tip due to gravity, though for thin spikes the shape of the water surface would be governed almost entirely by surface tension.

\subsection{What causes the flooding?}

Observationally, the main alternative to spike formation is flooding, so curiosity leads one to ask why flooding occurs, especially in view of the fact that it does not occur on the spikes themselves. Spike formation and flooding at the flat ice surface cannot occur at once, because there would be no pressure head to push the water up the spike. Spikes do form occasionally after flooding, as noted above, but flooding at the end of a spike was never seen, even when the water-iceair junction was on a vertical surface (e.g. Figs 7 and 10). (However, mechanical disturbance certainly could cause it to occur.) Why flooding occurs appears to be a difficult question. As discussed above, it is clear that some non-zero value of an advancing contact angle needs to be achieved before the flooding can start. However, if the freezing causes the junction line to follow the water-air interface, as appears to be the case, the contact remains at $0^{\circ}$ and flooding might never occur.

The immediate cause for starting a flood is very likely to be a mechanical disturbance, but in some circumstances flooding appears to be much easier to start than in others. For instance, situations like that illustrated in Figure 2, 
$t=2: 00$, with water bulging up above a number of large openings in the freezing surface, appear to be highly unstable: much more so than the water caps on growing spikes, even when the ice-water-air junction is vertical or overhanging. (Furthermore, more than gravity is pushing the water to flood downward in the cases with approximately hemispherical caps: the capillary pressure within a water drop is twice that within a water cylinder of the same radius.) Perhaps the higher curvature of the water surface at the end of a narrow spike stabilizes the tip of the spike against flooding, by making the water surface more resistant to deformation.

One way of thinking about the problem of flooding and its onset is to point out that if the water extrusion rate is too much faster than the growth rate of the ice, flooding must occur, but it is not known how much 'too much' is. 'Too much' certainly applies if the junction does not move at all, because the contact angle at the ice-air interface increases steadily as the water extrudes. Whenever it exceeds the 'effective advancing contact angle', flooding starts. For slow ice growth rates and fast extrusion rates, perhaps the critical factor is the concave radius of curvature of the ice surface at the junction. It becomes energetically favorable to fill in the curved ice surface with liquid that itself has a less curved surface. Then the common occurrence of flooding when the ice skim has a vertical $c$ axis would be due to the fact that growth in this crystal orientation decreases the area of open water surface the fastest. Almost all the surface will be icecovered before the water bulges very far above it.

There appear to be no studies on this subject, and an experimental investigation on it would be a major undertaking on its own. Results might depend critically upon surface purity and somewhat upon crystal orientation, and would probably be especially dependent upon thermal conditions at the water-ice-air junction. A referee of this paper pointed out a very interesting reference on the flooding of a solid by its own melt (Schiaffino and Sonin, $1997 \mathrm{a}, \mathrm{b})$, but the issue of the contact angle of water on ice may be more directly relevant here. A few of the studies that deal with it are Knight (1971), Elbaum and others (1993) and Makkonen (1997), but it becomes entangled with 'surface melting' on ice at temperatures below freezing, and present evidence does not seem to justify a specific value.

\subsection{Conclusions}

The curiosity that motivated this study was sparked by reviewing the papers by Hill and others (2004) (HLS) and Libbrecht and Lui (2004) (LL). Until then the author had seen ice spikes many times, because of habitually growing ice cubes for laboratory ice baths using pure water. He had assumed that the mechanism of the 'Bally-Dorsey hypothesis' (not knowing of its previous existence) was correct, and had not thought of any difficulties in understanding the phenomenon. As noted above, the first question that arose was why they were rare, given the rather obvious formation mechanism, and investigating that has led to other questions.

Perhaps the biggest difference between this study and both HLS and LL is its emphasis upon qualitative interpretations of direct, visual observations of the phenomenon, accompanied by measurements of its crystallographic aspects, whereas both HLS and LL started with measurements of frequency of spike occurrence as a function of growth conditions. The results here suggest that whether a spike occurs or not probably depends more on how the ice started in the ice-cube tray than on the physical conditions of freezing, though it certainly depends upon both. To the extent that that is true, the dependence of spike formation upon the controlled external conditions (temperature, wind, container properties and the like) could be indirect and quite obscure, through subtle influences on when, where and at what orientations the first ice crystals initiate. (Incidentally, the word 'nucleate' is purposely not used because true heterogeneous nucleation probably is involved rarely if at all in the formation of ice-cube spikes.)

This study has resulted in three main advances in understanding the physics of ice-cube spikes. First is an appreciation of the important role crystal orientations play in controlling whether spikes do or do not form. HLS suggested that ice-spike formation might be 'dominated by crystallographic effects', and the present results certainly underscore their importance. Hallett (1960) noted a probable influence of crystal orientation on the tilt of ice spikes, but was not clear on how that influence would operate. Second is a full appreciation of the necessary role of the effective, non-zero contact angle of water on ice in allowing spikes to form, the role of flooding as an alternative to spike formation, and the substantial lack of understanding of the criteria for flooding. Both LL and HLS mention it, but neither grant it a major role nor discuss the criteria for it. Third is an emphasis on understanding the steady state (or nearly steady state) that must exist at the tip of a growing spike that is growing with parallel or nearly parallel sides. (As noted in the introduction, a nearly constant diameter is a requirement by definition for a spike in this paper, and probably is a property of nearly all spikes in LL and HLS as well.) LL does discuss this, but not in depth.

While this study has largely satisfied the writer's curiosity about what is important in ice-spike formation, there are aspects of the phenomenon that are not well understood. Prominent among these are the criteria for the onset of flooding; the mechanism of the effect of salt upon flooding and its onset; and why spikes do not form when the $c$ axes of the crystals bounding the last open water are horizontal or close to horizontal. The temperature dependence of the frequency of ice-spike formation uncovered by LL and HLS remains a problem, which may or may not find an answer in the way the crystals initiate rather than the physical conditions of spike growth.

There may not be strong practical reasons for understanding ice spikes in great detail, but they remain an intrinsically interesting phenomenon with some puzzling aspects, and they are quite accessible to experiment. A major difficulty for experimentation is isolating the conditions needed for spike initiation from those needed for spike growth, because it is not entirely clear in which stage the crystal orientation relationships are selected. A continuation of this investigation past the exploratory stage might involve controlling the starting crystal fabrics by performing the confined freezing experiments in containers made of ice, so that the crystal orientations in the container walls determine the orientations bounding the last opening as the ice grows across the water surface. This could be done in practice quite simply, I think, by growing coarsegrained ice blocks, cutting out chunks that include either triple grain intersections or single grain boundaries with selected orientation relationships, drilling shallow holes into those chunks, and using those as the containers for the 
confined freezing. It should be possible to suppress initiation of new crystals entirely, and this should allow truly reproducible production of ice spikes; but one does not know this until one tries it. It would make an appealing thesis topic.

\section{ACKNOWLEDGEMENT}

The US National Center for Atmospheric Research is sponsored by the US National Science Foundation.

\section{REFERENCES}

Bally, O. 1933. Über eine eigenartige Eiskrystallbildung. Helv. Chim. Acta, 18, 475-476.

Blanchard, D.C. 1951. A verification of the Bally-Dorsey theory of spicule formation on sleet pellets. J. Meteorol., 8(4), 268-269.

Dorsey, H.E. 1921. Peculiar ice formations. Phys. Rev., 18(2), 162-164.

Elbaum, M., S.G. Lipson and J.G. Dash. 1993. Optical study of surface melting on ice. J. Cryst. Growth, 129(3-4), 491-505.

Hallett, J. 1960. Crystal growth and the formation of spikes in the surface of supercooled water. J. Glaciol., 3(28), 698-704.
Hill, L., E. Lozowski and R.D. Sampson. 2004. Experiments on ice spikes and a simple growth model. J. Glaciol., 50 (170), 375-381. Hobbs, P.V. 1974. Ice physics. Oxford, Clarendon Press.

Knight, C.A. 1971. Experiments on the contact angle of water on ice. Phil. Mag., 23, 153-165.

Langway, C.C., Jr. 1958. Ice fabrics and the universal stage. SIPRE Tech. Rep. 62.

Libbrecht, K.G. and K. Lui. 2004. An investigation of laboratorygrown 'ice spikes'. J. Glaciol., 50(170), 371-374.

Macklin, W.C. and B.F. Ryan. 1968. Growth velocities of ice in supercooled water and aqueous sucrose solutions. Phil. Mag., 17, 83-87.

Makkonen, L. 1997. Surface melting of ice. J. Phys. Chem. B, $101(32), 6196-6200$.

Perry, H.F. 1995. 'Last word' on ice spikes. Physics Teacher, 33(3), 148-149.

Pruppacher, H.R. 1967. On the growth of ice crystals in supercooled water and aqueous solution drops. Pure and Appl. Geophys., 68(1), 186-195. (10.1007/BF00874894.)

Schiaffino, S. and A.A. Sonin. 1997a. Motion and arrest of a molten contact line on a cold surface: an experimental study. Phys. Fluids, 9(8), 2217-2226.

Schiaffino, S. and A.A. Sonin. 1997b. On the theory for the arrest of an advancing molten contact line on a cold solid of the same material. Phys. Fluids, 9(8), 2227-2233.

MS received 27 September 2004 and accepted in revised form 2 February 2005 